\title{
Multilayers with tailored blurred interfaces
}

\author{
J. Padiyath, ${ }^{\text {a) }}$ J. Stahn, and M. Horisberger \\ Laboratory for Neutron Scattering, ETHZ \& PSI, 5232 Villigen, Switzerland \\ P. Böni \\ Physics Department E21, TUM, James-Frank Str., 85748 Garching, Germany
}

(Received 8 May 2006; accepted 3 August 2006; published online 15 September 2006)

\begin{abstract}
Monochromators used in neutron or x-ray experiments often have higher-order contributions, which have to be reduced by filters. Multilayer monochromators produced by dc-magnetron sputtering, with intentionally blurred interfaces, have reduced these higher-order contributions. The approach is to substitute the bilayer structure by several layers with individual thicknesses per period. A Ni/Ti monochromator was prepared where higher-order contributions were reduced below $0.1 \%$ of the first-order reflectivity of $93 \%$ (measured up to the seventh order). In order to determine the lifetime of such a structure, the interdiffusion of the constituents was measured with thermal annealing (interface diffusion activation energy: 0.16(1) eV). () 2006 American Institute of Physics.
\end{abstract}

[DOI: $10.1063 / 1.2355364]$

Monochromators made of single crystals are the most popular choice to select a certain wavelength in neutron or $\mathrm{x}$-ray scattering experiments. ${ }^{1,2}$ Beside this, especially for the experiments related to neutron reflectometry, monochromators based on multilayers play an important role. $^{3-5} \mathrm{~A}$ multilayer can be regarded as a one-dimensional single crystal with the lattice constant given by the adjustable multilayer period $d$ perpendicular to the surface. Experiments to produce artificial multilayers were performed as early as in 1939 using gold and copper as layer materials. ${ }^{6}$ Due to their high scattering length density contrast for neutrons, often $\mathrm{Ni}$ and $\mathrm{Ti}$ are selected as layer materials nowadays. They were widely investigated with respect to the multilayer interfaces. ${ }^{7-11}$ One of the major problems in realizing a truly monochromatic beam is higher-order reflections from multilayers as well as from single crystals. In the present case a unique approach to suppress higher-order reflections is given. By dividing the bilayers (two layers per period) of a multilayer monochromator into several layers with individual thicknesses, a quasisinusoidal scattering length density profile can be obtained, leading to a fundamental monochromator (only the first-order Bragg peak has significant intensity). ${ }^{12}$ Figure 1 is an illustration of this idea. A disadvantage is that the number of periods has to be increased by a factor of $\pi / 2$ if the scattering potential is changed from ideal sharp to sinusoidal interfaces.

The layers were deposited on $\mathrm{Si}$ (100) wafers using $\mathrm{Ar}$ ion dc-magnetron sputtering with no intentional heating. In order to ensure the homogeneity of the individual layers, the substrates were mounted on a moving table underneath the sputtering target. Small slits (width set to $12 \mathrm{~mm}$ ) were placed in front of the targets to reduce the sputtering rate, allowing for the deposition of very thin layers down to $2 \AA$. The deposition was carried out with only Ar gas at 50 SCCM (SCCM denotes cubic centimeter per minute at STP) (pressure within the chamber of $3 \times 10^{-3} \mathrm{mbar}$ ). A series of six multilayers has been prepared with a different number of layers per period $(2,6,10,14,18$, and 22) but the same nominal period. One additional sample was a fundamental

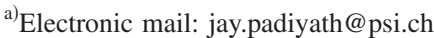

monochromator used as a filter at the neutron reflectometer NARZISS at SINQ, PSI Switzerland.

To verify the validity of the approach, specular and offspecular neutron reflectivity measurements have been performed on the neutron reflectometer ADAM (fixed wavelength of $4.4 \AA$ ) at the ILL in France and on AMOR (timeof-flight mode ${ }^{13}$ ) at SINQ in Switzerland. ${ }^{14} \mathrm{X}$-ray diffraction (XRD) experiments have been performed with $\mathrm{Cu} K_{\alpha}$ radiation (1.54 $\AA$ ). Transmission electron microscopy (TEM) has been used to investigate directly the layer structure using a Philips CM30ST with a voltage of $300 \mathrm{kV}$. The pointto-point resolution was $1.9 \AA$. The sample preparation technique for the measurements was ion etching with Ar ions at an angle of $4^{\circ}$ and with a voltage of $4.3 \mathrm{kV}$.

With respect to the higher-order contributions, the intended reduction has been obtained for an increasing number of layers per period. In Fig. 2 the neutron reflectivity of a fundamental multilayer monochromator with 22 layers per period of $14 \mathrm{~nm}$ and with 75 periods is presented, measured on the reflectometer ADAM. A reduction of higher-order reflections up to the seventh order is achieved, while maintaining a good reflectivity of $93 \%$ at the first-order Bragg peak. The rocking curve intensity (dotted line) shows a low background. Conventional monochromators (e.g., single crystals)

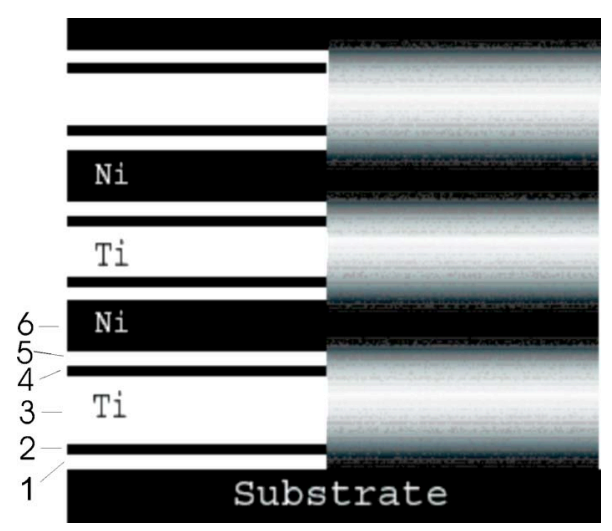

FIG. 1. Scheme of the multilayer cross section as sputtered on the left side with six layers per period with sharp interfaces. The right side shows blurred interfaces as expected for 22 layers per period. 


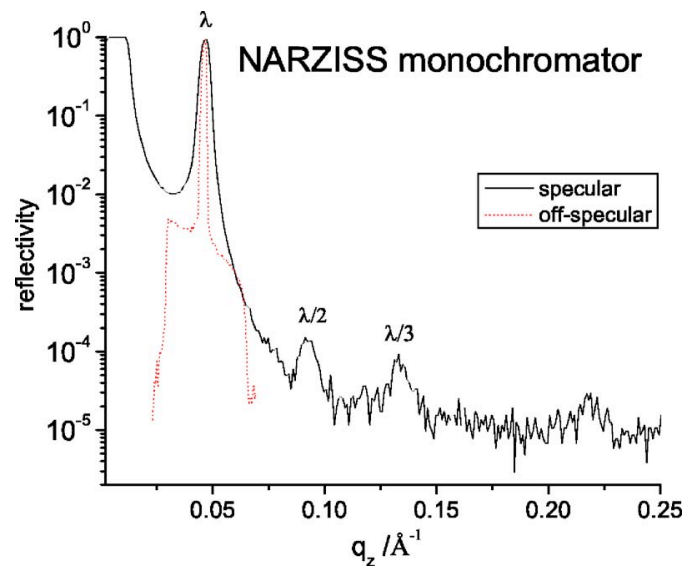

FIG. 2. (Color online) Neutron reflectivity of a fundamental multilayer monochromator with 75 periods. The measurements were performed using neutrons with a wavelength of $4.74 \AA$. The higher orders are significantly reduced. The rocking curve intensity (dotted) shows a low background.

might have reflectivities of $\approx 100 \%$. But the higher orders have to be reduced with, e.g., $\mathrm{BeO}$ filters, which reduce the neutron intensity down to $\approx 80 \%$. Figure $3(\mathrm{a})$ shows the offspecular neutron reflectivity of the bilayer. The Bragg sheets and Yoneda wings indicate a roughness of $4 \AA$. The higherorder contributions are observable up to the fourth order. In Fig. 3(b) the results of the fundamental monochromator are presented. The higher-order contributions vanish and the Bragg sheets are negligible.

TEM images of a bilayer and of a fundamental monochromator are shown in Fig. 4. While in the bilayer case sharp interfaces are visible, the individual layers could not be resolved in the latter case.

Electron diffraction, shown in Fig. 5, indicates a reduction in grain size for the fundamental monochromator and that there the grains are randomly oriented, while in the bilayer sample, the out-of-plane orientation of the grains is distributed approximately $\pm 10^{\circ}$ around the $\mathrm{Ni}$ and Ti peaks.

To achieve the same scattering potential profile with less layers (which are time consuming to produce), annealing was used to enhance the diffusion. This ansatz was applied earlier to bilayer samples to simulate aging or to reduce roughness. The first-order Bragg peak intensity is reduced in all annealed multilayers as expected. But in the case of the fundamental multilayer, there is an increase in the higher-order reflections (second and third-order Bragg peaks) after annealing at $523 \mathrm{~K}$. The growth of crystallites at the interfaces gives rise to sharper interfaces and therefore intensity gain in the higher-order contributions. To investigate the grain growth, x-ray diffraction experiments have been conducted before and after annealing. The grain size was calculated by
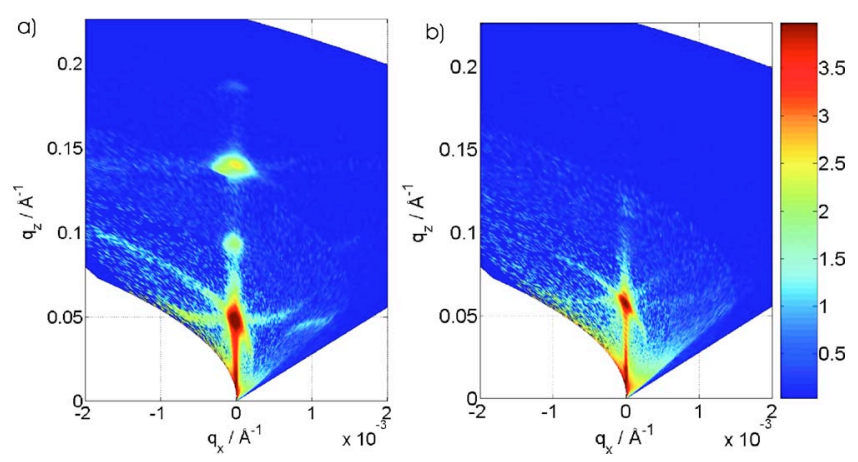

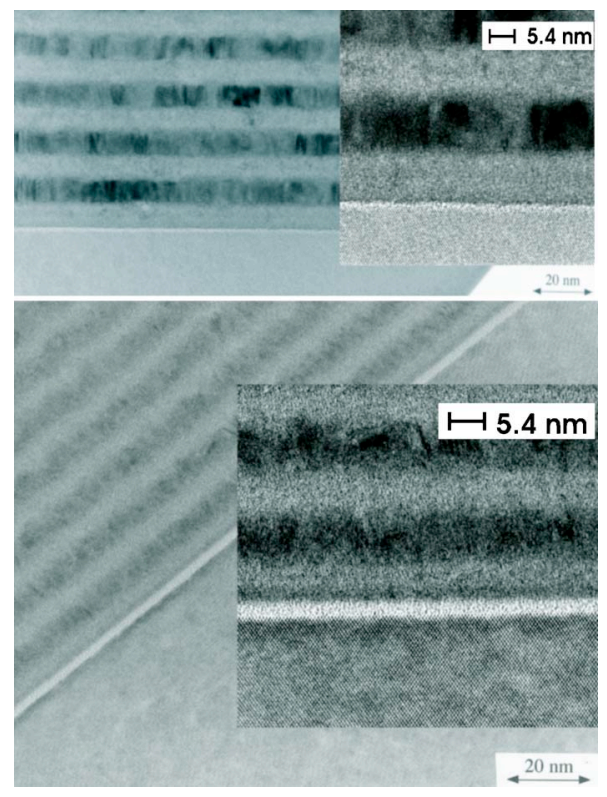

FIG. 4. TEM images of the bilayer (top) and the fundamental monochromator (bottom). The interface roughness is in both cases the same. In the case of the bilayer samples the crystallites are clearly visible, while in the case of the fundamental monochromator the thinner layers cannot be resolved.

the Scherrer formula. ${ }^{15}$ In a bilayer sample $4.0(3) \mathrm{nm}$ is obtained from the width of the $\mathrm{Ni}$ (111) peak. In the case of the sample with 22 layers per period, the as-deposited sample has a grain size (Ni 111) of 1.84(5) $\mathrm{nm}$ which grew during the annealing at $523 \mathrm{~K}$ to $2.9(1) \mathrm{nm}$.

Diffusion experiments have therefore been performed on the neutron reflectometers ADAM and AMOR, which gave information about the diffusivity and diffusion length. To obtain this information, a bilayer ([Ni $\left.16 \mathrm{~nm} / \mathrm{Ti} 8 \mathrm{~nm}]_{7}\right)$ has been annealed in situ at 293 (as prepared), 323, 373, 423, 473 , and $523 \mathrm{~K}$. By using the intensity of the second-order and fourth-order Bragg peaks, which are decreasing with increasing temperature, the diffusion coefficient $(D(T))$ has been calculated by means of ${ }^{16,17}$

$$
\frac{d}{d t} \ln \left(\frac{I(t)}{I_{0}}\right)=-\frac{8 \pi^{2} n^{2}}{d^{2}} D(T)
$$

where $I_{0}$ is the Bragg peak intensity before the annealing $(t=0), d$ the bilayer period, $n$ the order of the Bragg peak, and $t$ the annealing time. The weight of the Bragg peak was determined after a "background subtraction" due to the Fresnel reflectivity by multiplying the data by a factor of $q^{4}$.

Observed is the activation energy $E_{A}=0.16(1) \mathrm{eV}$ and for the pre-exponential factor $D_{0}=7 \times 10^{-21 \pm 1} \mathrm{~m}^{2} / \mathrm{s}$. $D_{0}$ is

FIG. 3. (Color online) Off-specular neutron reflectivity of the bilayer (left) and of the fundamental monochromator (right). For the bilayer the Bragg sheets and the Yoneda wings correspond to an interface roughness of $4 \AA$. In the case of the fundamental monochromator the Bragg sheets and thus the roughness is strongly reduced. 


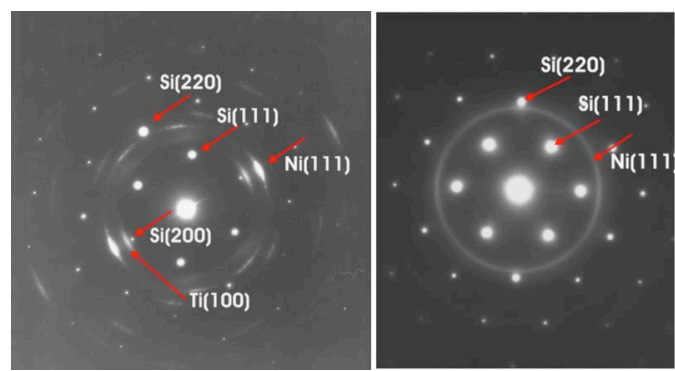

FIG. 5. (Color online) Electron diffraction measurements of the bilayer (left) and the fundamental monochromator (right). In the bilayer, the crystallites are oriented $\pm 10^{\circ}$ around $111 \mathrm{Ni}$ (fcc) and $100 \mathrm{Ti}$ (hcp). The right picture tells that the $\mathrm{Ni}$ crystallites are smaller and randomly oriented in the fundamental monochromator.

similar to earlier measurements by Boukhi et al. ${ }^{8}$ and Hollanders et al. with $\mathrm{Ni} / \mathrm{Ti}$ sputtered multilayers ${ }^{18}$ (calculated between $7.1 \times 10^{-21}$ and $11.3 \times 10^{-23} \mathrm{~m}^{2} / \mathrm{s}$, measured at 1 $\times 10^{-21} \mathrm{~m}^{2} / \mathrm{s}$ ). A very low activation energy leads to a larger diffusion length during the annealing process.

This explains the intensity gain for the higher-order reflections in the fundamental multilayer by facilitating the growth of small crystallites during the annealing process, which is confirmed by XRD measurements. Magnified parts in the case of the bilayer sample presented in Fig. 4 show grains of the order of $3 \mathrm{~nm}$ as in XRD experiments.

Summarizing, the presented approach to produce tailored blurred interfaces in multilayers was applied to create a fundamental monochromator, i.e., a periodic multilayer with a quasisinusoidal scattering potential. All higher-order Bragg reflections show at most less than $0.1 \%$ of the intensity of the first-order Bragg peak. Together with the reflectance of $93 \%$ this means that a very powerful device for monochromatization is obtained, which does not demand for an additional wavelength filter (as, e.g., cooled $\mathrm{BeO}$ filter). Further, it can be used in a broad wavelength range. Annealing experiments of a bilayer and of a fundamental monochromator showed the growth of crystallites in the thicker layers which was hindered during the deposition process by the thinner layers in the fundamental monochromator. The crystallites in the fundamental monochromator are randomly oriented within the layers and are growing during the annealing process from
1.8(1) to 2.9(1) $\mathrm{nm}$. The corresponding diffusion constant was found in the case of a $\mathrm{Ni} / \mathrm{Ti}$ bilayer with a very low activation energy of $0.16(1) \mathrm{eV}$.

The authors thank H. Grimmer (PSI) for his help with the x-ray experiments, E. Müller of the ETHZ for the TEM experiments and the discussion of the results, and M. Wolff for the assistance of the experiments on ADAM. Financial support by the Swiss National Science Foundation is gratefully acknowledged (200021-101567) and by MaNEP (NCCR). This work was supported by the European Union within the Sixth Framework Program FP6 under Contract No. 505925. This work was performed at the SINQ, Paul Scherrer Institute, Villigen, Switzerland and the ILL, Grenoble, France.

${ }^{1}$ P. Mikula, J. Kulda, P. Lukas, M. Vrana, and V. Wagner, Nucl. Instrum. Methods Phys. Res. A 338, 18 (1994).

${ }^{2}$ H. Maier-Leibnitz, Ann. Acad. Sci. Fenn., Ser. A6 267, 1 (1967).

${ }^{3}$ Neutrons in Biology, Basic Life Sciences Vol. 27, edited by B. P. Schoenborn (Plenum, New York, 1976), 125.

${ }^{4}$ A. A. van Well, V. O. de Haan, H. Fredrikze, and D. Clemens, Physica B 283, 282 (2000).

${ }^{5}$ A. K. Freund, Nucl. Instrum. Methods 124, 93 (1975).

${ }^{6}$ J. DuMond and J. P. Youtz, J. Appl. Phys. 11, 357 (1940).

${ }^{7}$ T. D. Shen, Y. L. Wu, M. X. Quan, J. T. Wang, and Z. Q. Hu, Mater. Lett. 17, 258 (1993).

${ }^{8}$ M. Bouhki, A. Bruson, and P. Guilmin, Solid State Commun. 83, 5 (1992).

${ }^{9}$ M. Bouhki, A. Bruson, P. Guilmin, Solid State Commun. 79, 389 (1991).

${ }^{10}$ M. A. Hollanders and B. J. Thijsse, J. Non-Cryst. Solids 117/118, 696 (1990).

${ }^{11}$ B. M. Clemens, Phys. Rev. B 33, 7615 (1986).

${ }^{12}$ J. Padiyath, J. Stahn, P. Allenspach, M. Horisberger, and P. Böni, Physica B 357, 218 (2005).

${ }^{13}$ M. Gupta, T. Gutberlet, J. Stahn, P. Keller, and D. Clemens, Pramana, J. Phys. 63, 47 (2004).

${ }^{14}$ W. E. Fischer, Physica B 234-236, 1202 (1997).

${ }^{15}$ B. D. Cullity, Elements of X-Ray Diffraction, 2nd ed. (Addison-Wesley, Reading, MA, 1978), p. 102

${ }^{16}$ J. Speakman, P. Rose, J. A. Hunt, N. Cowlam, R. E. Somekh, and A. L. Greer, J. Magn. Magn. Mater. 156, p 411 (1996).

${ }^{17}$ M. Gupta, A. Gupta, J. Stahn, M. Horisberger, T. Gutberlet, and P. Allenspach, Phys. Rev. B 70, 184206 (2004).

${ }^{18}$ M. A. Hollanders, B. J. Thijsse, and E. J. Mittenmeijer, Phys. Status Solidi A 127, 133 (1991). 